\title{
Table des matières
}

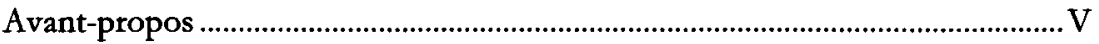

Table des matières ...............................................................................................

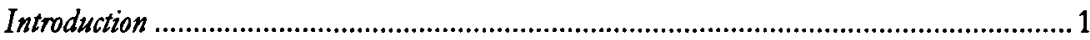

1. Peut-on lire le livre d'Isaie ?..............................................................................

1.1. Quelques remarques préliminaires..........................................................

1.2. A travers l'histoire de l'interprétation......................................................

1.3. Deux approches pour une (re)découverte du livre d'Isaïe ....................5

1.3.1. Un intérêt relativement récent pour l'unité du livre...................5

1.3.2. Les deux approches sont-elles compatibles ? .............................. 7

1.3.3. Principes de base fournis par la Redaktionsgeschichte............. 9

2. Recherche d'un principe unificateur................................................................11

2.1. A l'enseigne de la justice......................................................................11

2.2. Structures d'ensemble..........................................................................13

2.3. Le livre d'Isaie: Une torah prophétique ? ............................................15

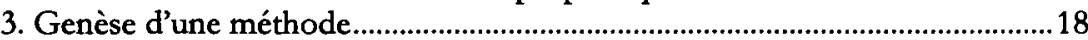

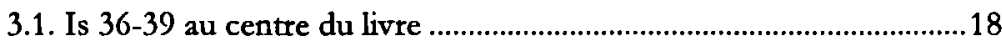

3.2. Isaïe et la maison royale ......................................................................... 19

3.3. L'oracle de Nathan comme fil rouge................................................2

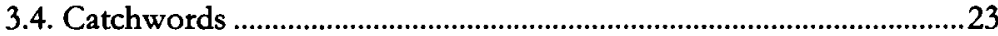

Panorama inaugural: Is 1,1-2,5

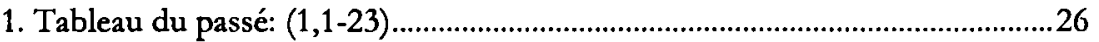

1.1. Titre et renvoi à l'historiographie biblique .........................................26

1.2. L'appel à témoins rappelle le Cantique de Moïse ..................................30

1.3. Les « dramatis person $x$ " ..............................................................................32

1.4. La protection divine et ses conditions .................................................33

1.4.1. Le salut accordé par Dieu.............................................................33

1.4.2. Vos mains sont pleines de sang......................................................34

1.5. Sion est descendue bien bas ......................................................................37

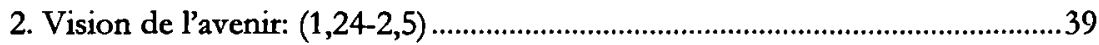

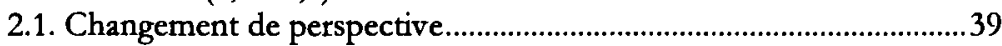

2.2. Le diptyque.......................................................................................41

2.3. Pourquoi un deuxième titre .................................................................43

2.4. La vision de la Maison de YHWH......................................................4

2.4.1. Restauration...............................................................................4

2.4.2. Dépassement ...........................................................................45 
2.4.3. Maison de YHWH et maison de Jacob ................................... 46

3. Leçons à tirer de la vision inaugurale .................................................................. 49

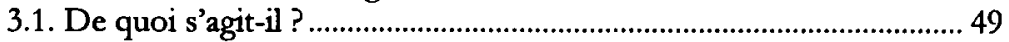

3.1.1. Genre littéraire …………………….............................................. 49

3.1.2. Portée du livre .......................................................................... 50

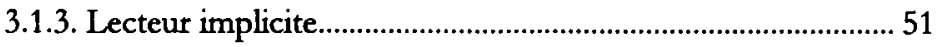

3.2. Comment lire ?.................................................................................. 52

3.2.1. La " maison ", ses bâtisseurs et ses habitants.......................... 52

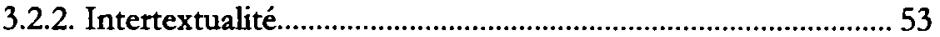

Le roi en sa maison: Is 2,6-6,13

1. Est-ce Dieu qui a abandonné la maison (2,6a) ? ................................................ 56

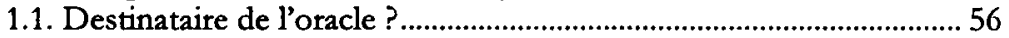

1.2. Le rapport de filiation .......................................................................... 57

1.3. Maison vide de Dieu et maison remplie de biens .............................. 58

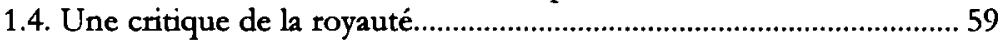

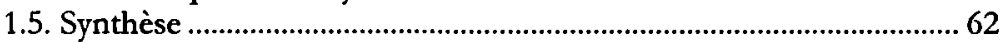

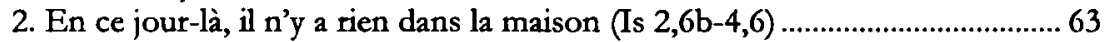

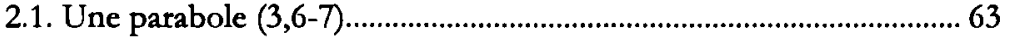

2.1.1. Maison habitée et maison vide de bien (a) ................................ 64

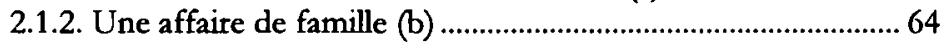

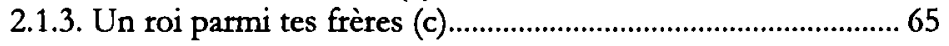

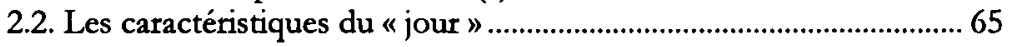

2.2.1. Première section: Enfermement d'Israël (2,6b-22) ................. 66

2.2.2. Deuxième section: Nettoyage par le vide $(3,1-4,1)$................. 67

2.2.3. Troisième section : Descendance $(4,2-4,6)$................................ 68

2.2.4. Synthèse.................................................................................... 70

3. La maison où YHWH est roi au milieu de sa vigne $(5,1-6,13)$....................... 73

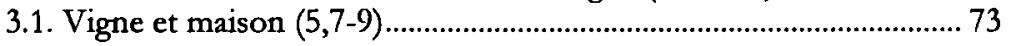

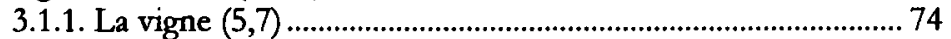

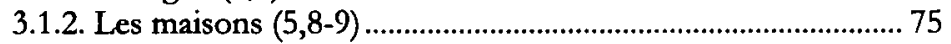

3.2. La vision du Roi $(6,1-13)$.............................................................. 76

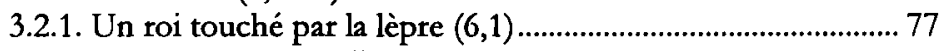

3.2.2. La maison se remplit $(6,4)$..................................................... 78

3.2.3. Jusqu'à ce que les maisons soient vides $(6,11) \ldots \ldots \ldots \ldots \ldots \ldots \ldots \ldots . . . . . . . .78$

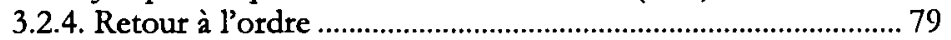

3.3. Autres éléments justifiant le découpage de la péricope $(5,1-6,13) \ldots 81$

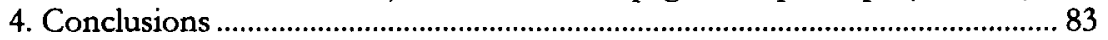

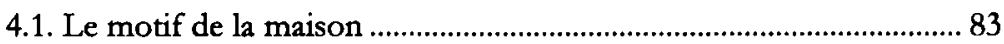

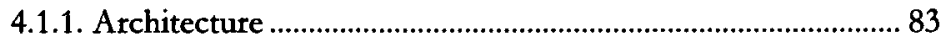

4.1.2. L'intrigue et ses enjeux............................................................... 84

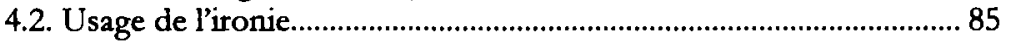


La maison de David et la fille de Sion: Is 7,1-12,6

1. Introduction .88

1.1. D'un livre à l'autre et d'une partie à l'autre ......................................89

1.1.1. Achaz, petit-fils d'Ozias........................................................89

1.1.2. Au temps des rois d'Israël et de Juda ..................................90

2. Le cœur de la maison de David $(7,2)$.............................................................92

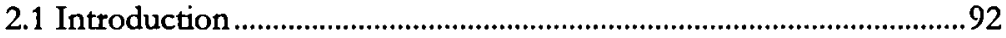

2.2. Maison de David et maison de YHWH (2 Sm 7)............................93

2.3. La « maison de David » dans le livre des Rois ....................................97

2.4. A qui va-t-on comparer le rejeton de la " maison de David "...........98

2.4.1. La « maison de David » en Is 7 et le roi David en $2 \mathrm{Sm} 7 \ldots . .98$

2.4.2. Achaz (Is 7,3) et Ezéchias (2 R 18,7)......................................99

2.5. Qui peut bénéficier des promesses faites à David ?.........................100

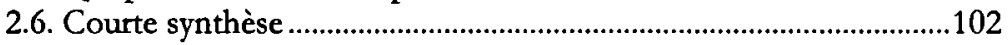

3. Quel avenir pour la maison de David $(7,1-9,6)$ ? ...........................................104

3.1. La maison de David, l'Emmanuel et le jour $(7,1-25) \ldots \ldots \ldots \ldots \ldots \ldots . . . . .104$

3.1.1. Shear Yashoub...........................................................................104

3.1.2. « Ecoutez, maison de David ! " ................................................105

3.1.3. Qui est l'Emmanuel ? ...........................................................105

3.1.4. Eclatement du cadre narratif et implication du lecteur........107

3.1.5. Des jours.................................................................................109

3.1.6. Synthèse et parallèle avec la première partie..........................110

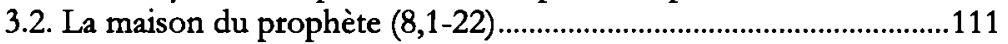

3.2.1. D'un chapitre à l'autre: du roi au peuple................................111

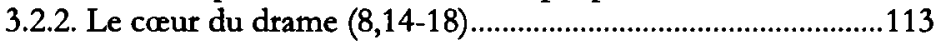

3.2.3. Correspondances avec la première partie .............................115

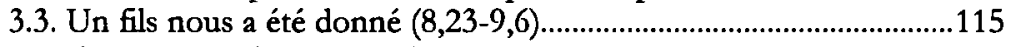

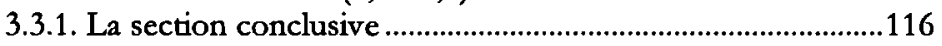

3.3.2. Une naissance royale .........................................................116

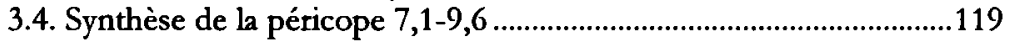

3.4.1. Le motif auxiliaire du « don des enfants ».............................119

3.4.2. Le motif de la « maison »......................................................120

3.4.3. Résumé de la péricope ...........................................................122

4. Menace et salut pour la « fille de Sion 》 ............................................................124

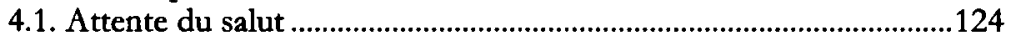

4.2. « Nous bâtirons en pierre de taille » $(9,7-10,4)$.................................125

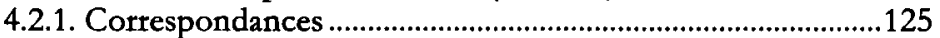

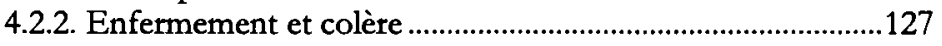

4.2.3. Le « peuple » et le lecteur..........................................................128

4.3. Le reste d'Israël et les rescapés de la maison de Jacob (10,5-34) ...128

4.3.1. La colère se retourne contre l'Assyrie ...................................128

4.3.2. La purification du peuple est inévitable ...............................130

4.3.3. Le prophète et le lecteur..........................................................133

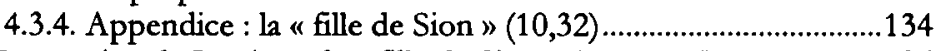

4.4. $\mathrm{La}$ « racine de Jessé » et la « fille de Sion » $(11,1-12,6) \ldots \ldots \ldots \ldots \ldots \ldots . . . .136$ 


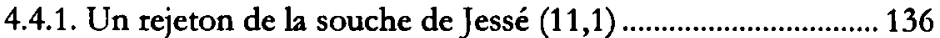

4.4.2. Sagesse et repos sur la montagne sainte $(11,9-10)$............... 139

4.4.3. Un nouvel Exode sans nouveau Moïse ?................................. 140

4.4.4. « Celle qui habite à Sion » ..................................................... 143

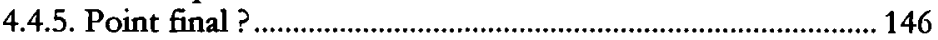

5. Conclusions

5.1. Le motif de la « maison » et le paysage textuel................................. 147

5.1.1. Les grandes articulations ..................................................... 147

5.1.2. Agencement des versets au sein d'une même section ......... 149

5.2. Le motif de la « maison » et la progression de l'intrigue................. 149

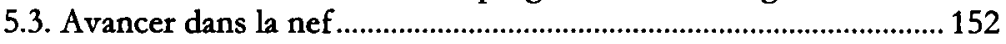

L'assaut des nations vers la maison du Seigneur: Is 13-23

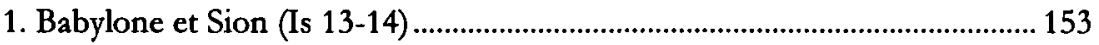

1.1. « Sur une montagne dénudée... » $(13,2)$........................................ 153

1.1.1. Echos des chapitres précédents ........................................... 153

1.1.2. Absence significative du motif de la maison.......................... 156

1.1.3. Implication du lecteur ............................................................ 156

1.2. L'étranger se joindra à eux pour s'associer à la maison de Jacob.. 158

1.2.1. Triple thématique...................................................................... 158

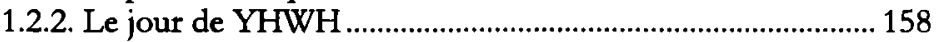

1.2.3. Le lecteur implicite............................................................. 159

1.3. Contre le roi de Babylone,...entre autres (14,4b-32) ..................... 159

1.3.1. Le paroxysme de l'orgueil....................................................... 159

1.3.2. Le roi de Babylone, privé de sépulture et de succession.... 160

1.3.3. YHWH a fondé Sion, en elle se réfugieront les pauvres..... 161

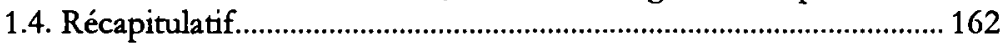

2. Les habitants du monde, ceux de Jérusalem et le Créateur (Is 15-23) ......... 163

2.1. La maison de Moab (15,2)............................................................... 163

2.1.1. Dernière balise................................................................... 163

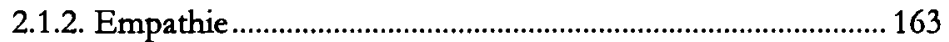

2.1.3. Triple thématique................................................................... 164

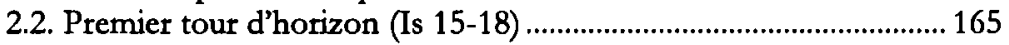

2.2.1. « Moab se lamente sur Moab »........................................... 165

2.2.2. « La gloire de Moab sera allégée \#........................................ 166

2.2.3. « Le lieu où réside le nom de YHWH »............................... 167

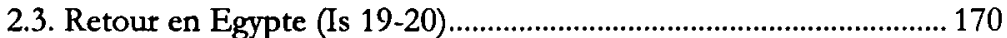

2.3.1. Voici que YHWH...vient en Egypte $(19,1) \ldots \ldots \ldots \ldots . . . . . . . . . . . . .170$

2.3.2. « Mon peuple l'Egypte " $(19,24)$............................................ 171

2.4. Conclusion : « la honte de la nudité de l'Egypte » (ch. 20) ............ 172

2.5. Les maisons dans le chaos (Is 21-22).............................................. 174

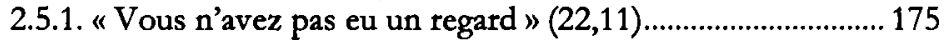

2.5.2. Le sort de la maison de David $(22,15-25)$............................. 176

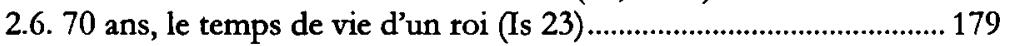

3. Conclusions 
3.1. Le motif de la maison ................................................................180

3.2. Présence de la vision inaugurale.......................................................181

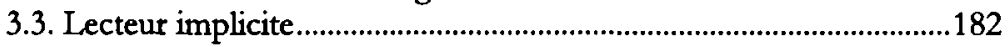

\section{Purification et salut pour l'babitant du monde et la cité de David : Is 24-33}

1. L'habitant du monde et le Roi de Jérusalem (Is 24-27) ...............................184

1.1. Les habitants sont dispersés car les maisons sont fermées (Is 24).184

1.1.1. Liens avec ce qui précède ........................................................185

1.1.2. Les habitants sont dispersés................................................185

1.1.3. La cité du tohu et la montagne de Sion...............................188

1.1.4. Le Roi de Jérusalem contre les rois de la terre ......................189

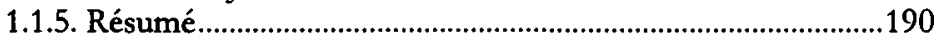

1.2. Célébration du salut de Dieu (Is 25-27) ..........................................190

1.2.1. Quelle habitation: « Tas de pierre » ou « montagne »............191

1.2.2. En « ce jour-là », fin de l'endurcissement..............................194

1.3. Courte synthèse ..................................................................................195

2. L'habitant de Jérusalem et le Roi du monde (Is 28-33) ..................................197

2.1. Une unité structurée par six malédictions ......................................197

2.2. Quels sont les destinataires de la harangue prophétique ?..............198

2.3. Présences du motif de la maison..........................................................200

2.3.1. La pierre angulaire et le refuge du mensonge......................200

2.3.2. Siège de Jérusalem ...............................................................201

2.3.3 Evolution et transformation de la Cité...................................202

2.3.4. Evolution et transformation de la maison de Jacob.............204

2.4. Qui est « le roi » du chapitre 32 ? ........................................................207

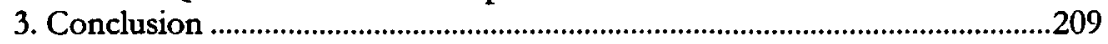

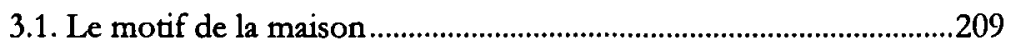

3.1.1. Balises et motifs auxiliaires................................................209

3.1.2. Triple thématique ...............................................................210

3.1.3. Unité du motif..........................................................................2. 210

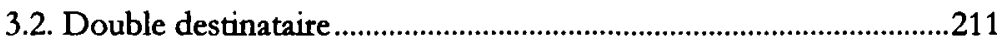

L'espérance renouvelée: Is 3446

1. Diptyque d'ouverture de la deuxième partie (Is 34-35) ................................214

1.1. Que la terre écoute avec tous ses descendants $(34,1-4) \ldots . . . \ldots . . . . . . . . .215$

1.2. Boçra est une demeure pour les filles de l'autruche $(34,5-17) \ldots \ldots . .216$

1.2.1. Edom et Sodome..............................................................216

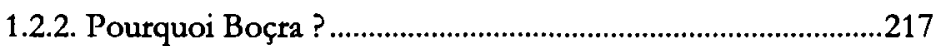

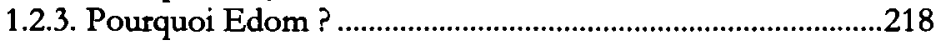

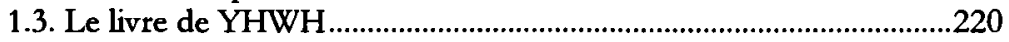

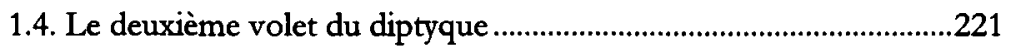

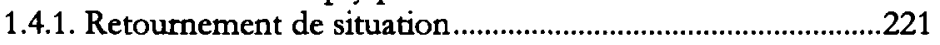

1.4.2. Parallèle avec le Panorama Inaugural ..................................222

1.4.3. Correspondance entre les deux parties du diptyque..............222 


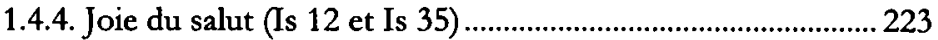

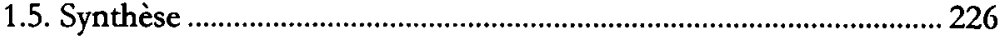

2. Le prophète Isaïe et la maison de David: suite et fin (Is 36-39) .................. 227

2.1. Où l'on retrouve Eliagim (Is 36) ......................................................228

2.1.1. Völkerkampf ou montée des peuples ..................................... 228

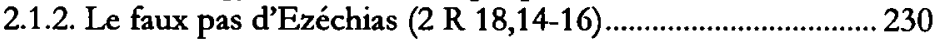

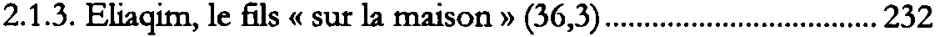

2.1.4. Le Ravshakeb joue les prophètes: oracles de jugement......... 233

2.1.5. Le Ravshakeh joue les prophètes: oracles de consolation .... 236

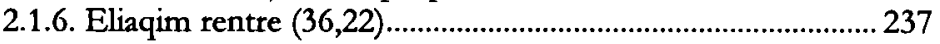

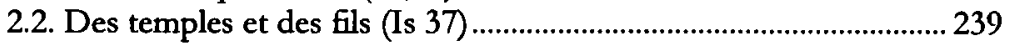

2.2.1. Le sort de la maison de David - suite $(37,1-13)$.................. 239

2.2.2. Une lettre dans la maison de YHWH (37,14-20) ................. 242

2.2.3. Confrontation entre Assur et la fille de Sion (37,21-35) .... 244

2.2.4. A cause de moi et à cause de mon serviteur David $(37,35) 246$

2.2.5. Sic transit Sennachérib $(37,38)$.............................................. 247

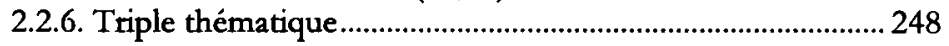

2.3. Un sursis pour la maison de David (Is 38) ..................................... 249

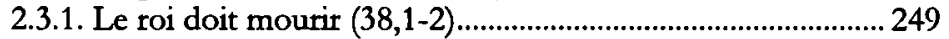

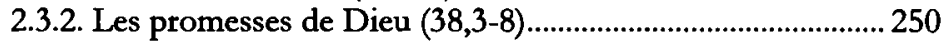

2.3.3. Ezéchias rend grâce pour avoir échappé au sort des rois ... 251

2.4. Comment Ezéchias conduisit les nations à sa maison (Is 39)....... 254

2.4.1. Le salut par la descendance royale ?.................................... 254

2.4.2. Les griefs accumulés contre la maison royale ...................... 255

2.4.3. La maison d'Ezéchias et le temple du roi de Babylone....... 256

2.4.4. Le sort de la maison de David: fin ?..................................... 258

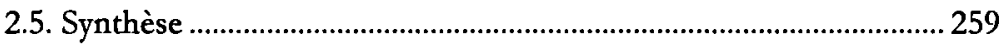

2.5.1. Le motif de la maison et le paysage textuel........................... 259

2.5.2. Le motif de la maison et la progression de l'intrigue........... 260

2.5.3. Implication du lecteur ................................................................ 261

3. Est-ce toi qui me bâtiras une maison (Is 40-45).............................................264

3.1. La voie de YHWH et l'espérance (partiellement) renouvelée........ 264

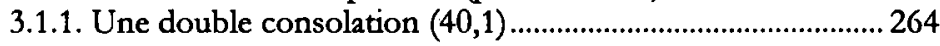

3.1.2. Investiture des disciples comme prophètes $(40,1-8) \ldots . . . . . . . .265$

3.1.3. La messagère de Sion $(40,9-11)$............................................... 266

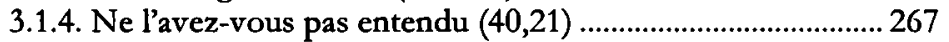

3.1.5. Il habite le dôme couvrant la terre $(40,22)$........................... 267

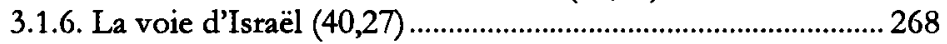

3.2. Le Roi de Jacob et son serviteur (Is 41-42) .................................... 270

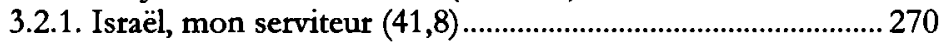

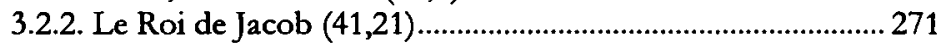

3.2.3. Qui était aveugle et sourd comme mon serviteur? (Is 42) . 271

3.3. Israël est sauvé par YHWH (Is 43-46) ............................................ 273

3.3.1. Ce n'est pas toi qui m'as formé, c'est moi qui t’ai formé... 273

3.3.2. Une nouvelle dynastie ............................................................. 275

3.3.3. Un nouveau temple à Jérusalem ........................................... 275 
Une maison de prière pour tous les peuples: Is 47-66

1. Je maintiendrai les bienfaits accordés à David (Is 47-55)................................278

1.1. Pas de sauveur pour Babylone (Is 47) ..............................................278

1.1.1. La fille de Babylone habitera la poussiète $(47,1) \ldots \ldots \ldots \ldots \ldots . . . . .278$

1.1.2. Babylone privée de ses enfants $(47,8-9)$...................................279

1.2. YHWH façonne son serviteur (Is 48-49) .........................................280

1.2.1. « Ecoutez ceci, maison de Jacob » $(48,1)$..............................280

1.2.2. D'un révolté, YHWH se fait un serviteur...............................281

1.2.3. Sion sera rebâtie par des fils $(49,14-26) \ldots \ldots \ldots \ldots \ldots \ldots \ldots \ldots \ldots \ldots \ldots \ldots . . .282$

1.3. Des fils appelés à devenir serviteurs (Is 50-55)..............................283

1.3.1. YHWH parle à ses fils et à leur mère $(50,1-52,12)$................283

1.3.2. Exaltation du serviteur $(52,13-53,12)$...................................285

1.3.3. Sion est reconstruite et ses fils abondent (Is 54)..................286

1.3.4. Confirmation et élargissement de l'alliance davidique .........287

1.4. Synthèse ...........................................................................................28

2. Mon salut est sur le point d'arriver (Is 56-66) ..............................................289

2.1. Une maison de prière pour tous les fils d'Adam $(56,1-8)$................289

2.1.1. Le salut proposé à tout fils d'Adam........................................289

2.1.2. L'eunuque et le fils de l'étranger dans la maison de prière..290

2.2. Une descendance spirituelle pour la maison de Jacob $(56,9-59,21) 292$

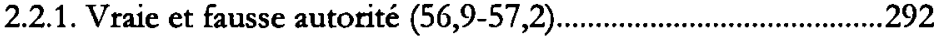

2.2.2. YHWH fait la paix avec les convertis $(57,3-21) \ldots \ldots \ldots \ldots \ldots \ldots . . .292$

2.2.3. Restauration de la maison de Jacob (Is 58-59) ......................294

2.3. Notre Père rassemblera tous ses fils en sa maison $(60,1-63,16) \ldots . .296$

2.3.1. La maison de ma splendeur (Is 60) ........................................296

2.3.2. Le messie et sa descendance (Is 61) .........................................297

2.3.3. La fille de Sion (Is 62) .........................................................298

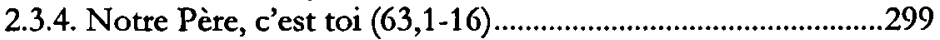

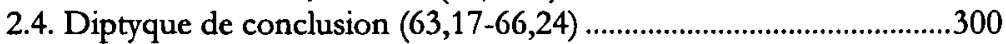

2.4.1. Notre maison a été embrasée par le feu $(64,10) \ldots \ldots \ldots \ldots \ldots \ldots . . . . .300$

2.4.2. « Mes serviteurs demeureront » sur ma montagne $(65,9)$....301

2.4.3. Quelle est donc la maison que vous bâtiriez pour moi ?......303

\section{Conclusion}

\section{Conclusion et ouvertures}

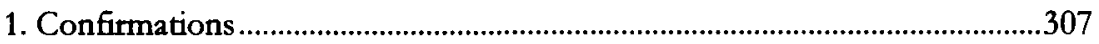

1.1. La vision de la totalité .........................................................................307

1.2. Un fonctionnement dialogique ..........................................................307

2. La maison du serviteur, héritière des promesses faites à David......................308

2.1. La construction d'une intrigue prophétique......................................308

2.1.1. Les fondements.........................................................................309

2.1.2. L'incapacité de la maison de David ........................................309 
2.1.3. Le " groupe du nous » au service du rassemblement........... 310

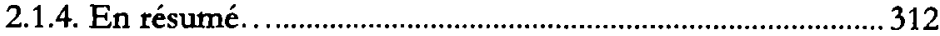

2.2. Le balisage textuel........................................................................ 312

2.3. La mise en évidence de structures contrastées ................................. 313

2.4. Le décryptage de l'ironie prophétique ............................................ 313

2.4.1. Contrastes................................................................................ 313

2.4.2. Entre revirement et retournement....................................... 313

2.4.3. Décryptage ........................................................................... 314

3. Le livre d'Isaie, celui des Psaumes et la lecture des livres bibliques ............. 316

3.1. Datation relative des deux ouvrages ................................................. 317

3.2. Des techniques... au service d'une pédagogie ?............................... 317

3.3. La postérité de l'oracle de Nathan ...................................................318

4. Lire la Bible comme un livre ? .................................................................. 320

Annexes

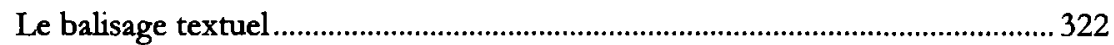

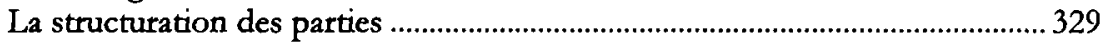

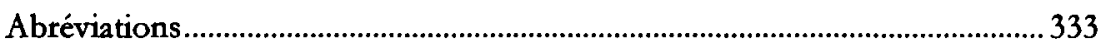

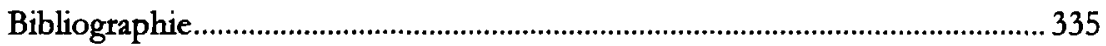

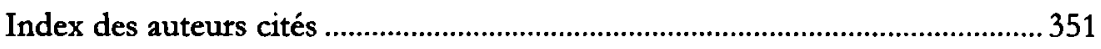

